\title{
The central role of SOCS-3 in integrating the neuro-immunoendocrine interface
}

\author{
Christoph J. Auernhammer ${ }^{1}$ and Shlomo Melmed ${ }^{2}$ \\ ${ }^{1}$ Department of Internal Medicine II, Klinikum Grosshadern, Ludwig-Maximilians-Universität, Munich, Germany \\ ${ }^{2}$ Cedars-Sinai Research Institute, UCLA School of Medicine, Los Angeles, California, USA
}

Address correspondence to: Shlomo Melmed, Academic Affairs, Cedars-Sinai Medical Center, 8700 Beverly Boulevard, Room 2015, Los Angeles, California 90048, USA.

Phone: (310) 423-4691; Fax: (310) 423-0119; E-mail: melmed@csmc.edu.

J. Clin. Invest. 108:1735-1740 (2001). DOI:10.1172/JCI200114662.

Cytokines and related signaling molecules lead to profound regulatory changes in differentiated cell function, modulating immune functions, the stress response, energy metabolism, growth, and reproduction. A network of intracellular molecules that dampen or inhibit the effects of these pleiotropic factors provides a crucial counterbalance to cytokine signals. Recent studies have shown that negative feedback, initiated in the various target tissues by the cytokines themselves, is central to endocrine homeostasis. Cytokine-mediated adrenocorticotropin hormone (ACTH) and cortisol overproduction, for instance, is prevented by tightly regulated cytokine-induced intracellular negative control systems. Likewise, growth hormone (GH) signaling is abrogated by cytokine-induced proteins, providing an explanation for $\mathrm{GH}$ resistance and stunted growth observed in states of elevated cytokine activity, including inflammation, starvation, and chronic illness. This article explores the role of the signal suppressor SOCS-3 in inhibiting the actions of neuro-endocrine cytokines and hormones, while maintaining the plasticity of the ultimate neuroimmune endocrine responses.

\section{SOCS proteins as inhibitors}

of the JAK-STAT cascade

The JAK-STAT cascade is an intracellular signaling pathway shared by a variety of cytokines, including gp130 cytokines (IL-6, IL-11, leukemia inhibitory factor [LIF], oncostatin M [OSM], ciliary neurotrophic factor [CNTF], cardiotropin-1 [CT-1], cardiotropin-like cytokine [CLC]), as well as leptin, $\mathrm{GH}$, and prolactin. Ligand binding to cytokine receptors, which themselves lack intrinsic kinase activity, activates receptor-associated Janus kinases (JAK's) by autophosphorylation and subsequent tyrosine phosphorylation of the receptor's cytoplasmic domain and of associated proteins termed STATs for signal transducers and activators of transcription. Tyrosine phosphorylation of STATs enables homo- or heterodimerization of various STAT proteins. The dimerized STAT complexes translocate to the nucleus, where they transactivate their target genes by binding to specific promoter elements $(1,2)$.
A family of proteins able to inhibit the JAK-STAT signaling cascade has synonymously been described as suppressor of cytokine signaling (SOCS) protein (3), JAK-binding protein (4), and STAT-induced STAT inhibitor. The SOCS protein family (5) encompasses SOCS-1 $(3,4,6)$, SOCS-2 (3), SOCS-3 (3), CIS (7), and SOCS-4 to SOCS-7 (5). While the role of the former four proteins is increasingly well understood, the roles of SOCS-4 to SOCS-7 remain poorly characterized. In vitro overexpression studies demonstrate that SOCS-1 and SOCS-3 exert similar effects and represent the most potent and broadly acting suppressors of cytokine signaling. These factors potently inhibit JAKSTAT signaling of several gp130 cytokines (see Arzt, this Perspective series, ref. 8; and refs. 3, 4, 9-12), GH (13-15), and prolactin (16).

The common protein structure of SOCS proteins is a variable $\mathrm{N}$-terminal region, a central $\mathrm{SH} 2$ domain, and a C-terminal domain, termed SOCS-box motif (5). The central SH2 domain alone is not sufficient for inhibiting JAK-STAT signaling (4), as part of the $\mathrm{N}$-terminal region, termed pre-SH2 domain/kinase inhibitory region, is also required $(10,17,18)$. The C-terminal SOCS-box interacts with elongin BC complex (19), although the physiological significance of this interaction is still unclear. Some observations suggest that this interaction protects SOCS proteins from degradation in the ubiquitin-proteasome pathway, whereas others suggest that it actually directs these proteins to the ubiquitin-proteasome pathway and sets the stage for their degradation $(19,20)$. Conversely, the significance of the proteasome in the turnover of these molecules is not in doubt. The halflives of SOCS- 1 and SOCS-3 in COS-7 cells have been calculated to be as short as 1.5 hours (21), but incubation with proteasome inhibitors stabilizes them significantly $(18,19)$. Therefore, the C-terminal SOCS-box also appears not to be essential for direct inhibitory interaction with JAK $(10,17)$ but might be required to control SOCS protein degradation and thus might modulate the intracellular level of the SOCS protein (18). 


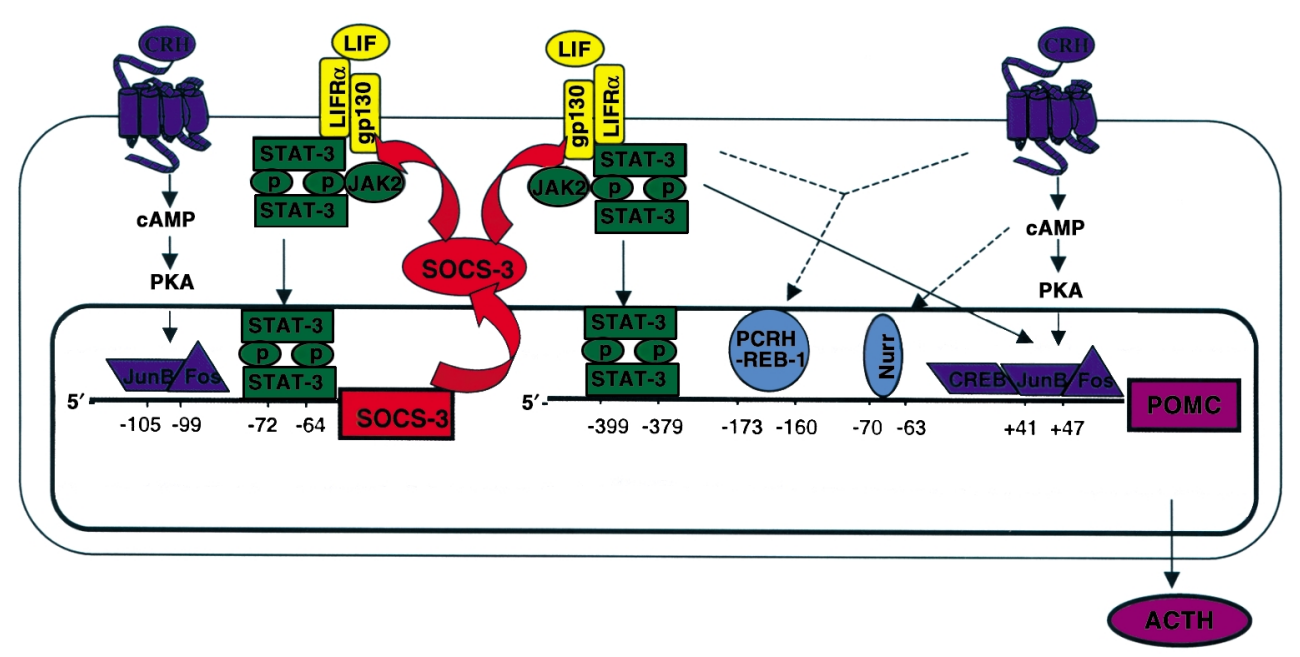

Figure 1

Corticotroph SOCS-3 as an intracellular suppressor of cytokine signaling. Corticotroph SOCS-3 inhibits STAT-dependent POMC gene expression by negatively interfering with LIF-induced activation of the JAK-STAT cascade. In contrast, CRH-induced POMC gene expression is not affected by SOCS-3. LIF stimulation of the corticotroph results in rapid upregulation of SOCS-3 by a STAT-dependent mechanism. Thus, LIF-induced activation of corticotroph POMC gene activation is limited by parallel induction of SOCS-3 expression, rendering the cell resistant to further JAK-STAT activation. On the other hand, autoregulation of STAT-dependent SOCS-3 gene expression and rapid degradation of SOCS-3 protein by the proteasome pathway enable the cell to restore its functional status. LIF and CRH synergistically induce POMC promoter activity. LIF activates POMC promoter activity not only by direct binding of activated STATs, but also indirectly by inducing STAT-dependent expression of transcription factors $\mathrm{c}$-fos and JunB. CRH also induces SOCS3 promoter activity by binding of c-fos and JunB. Thus, CRH indirectly inhibits LIF-induced POMC promoter activation and downregulates the synergistic cross-talk of CRH and LIF on POMC promoter activity. Adapted from ref. 34 and ref. 47 with permission.

SOCS-1 and SOCS-3 suppress JAK-STAT signaling by similar mechanisms. SOCS- 1 associates with and inhibits JAK1 (4, 22), JAK2 (4, 6, 15-17), JAK3 (4, 22), Tyk 2 (4), and Tec (6). Following early autophosphorylation of Tyr1007 in the JH1 domain of JAK2 $(4,17)$, SOCS- 1 binds via its SH2 domain to the catalytic JH1 domain of JAK2 (4), thus inhibiting JH1 activity. SOCS-3 also coimmunoprecipitates with JAK2 (18, 23 ) and binds Tyr1007. SOCS-3 is distinguished from SOCS- 1 by its lower affinity for JAK2 (16). In addition to direct JAK interaction, SOCS-3's action is also mediated by binding to phosphorylated tyrosine residues in the intracellular domain of various receptors - Tyr759 of gp130 (24), Tyr333 and Tyr338 of GH receptor (15), or Tyr985 and Tyr1077 of the long receptor of leptin $(25,26)$ - which allows it to suppress receptor function directly as well as indirectly. Similarly, CIS has been demonstrated to exert its inhibitory role by binding to tyrosine residues of intracellular receptor domains (7).

Despite in vitro overexpression data demonstrating similar suppressor activities for SOCS-1 and SOCS-3, these proteins are induced specifically by different cytokines and in different cell types, suggesting that they play different roles in vivo. Induction of SOCS expression by various cytokines is STAT-dependent, and indeed, the promoter regions of the cis $(7,27)$, SOCS1, and SOCS3 genes (28) show functional STAT-binding elements. Cytokine-induced expression of SOCS-1 and SOCS-3 can be inhibited by STAT3 dominant negative mutants (28), while gene expression of CIS can be inhibited by STAT5 dominant negative mutants (29).
LIF, a modulator of hypothalamic-pituitary-adrenal axis function

LIF is a potent neuro-immunoendocrine modulator of pituitary corticotroph function in vivo and in vitro. Both alone and in additive or synergistic actions with $\mathrm{CRH}$, LIF induces proopiomelanocortin (POMC) gene expression and ACTH secretion by cultured murine $(30,31)$ and human (32) corticotrophs. In these cells, LIF stimulates the JAK-STAT signaling cascade, causing phosphorylation of JAK2 (18), gp130 (33), STAT3 $(31,33,34)$, and STAT1 (31). LIF-induced POMC gene expression is critically STAT3-dependent, as dominant negative STAT3 mutants significantly decrease LIF-induced POMC promoter activity and gene expression (34).

Increase of POMC promoter activity following STAT3 activation is mediated by direct and indirect mechanisms (35). The rat POMC promoter region contains two juxtaposed STAT3-binding elements (-399 TTTACCTCCAAATGCCAGGAA- -379), their sequences only distantly related to the classic STAT3 consensus DNA-binding sequence (TTCCA). While each of these elements can bind STAT3, both are required for highaffinity binding (35). Mutation of the STAT3 DNAbinding sequence in this region reduces LIF-induced POMC promoter activity by half. In addition, STAT3 (either alone or in synergy with $\mathrm{CRH}$ ) can stimulate $P O M C$ promoter activity indirectly by stimulating c-fos and JunB expression (35), thus allowing the formation of the Fos/Jun heterodimer, AP-1 (36). Interestingly, the genes for each of these transcription factors harbor STAT-binding elements in their promoters. In addition, binding of $\mathrm{c}$-fos and JunB to an AP-1 site in the 
POMC exon 1 occurs in vitro and appears to participate in LIF-induced transactivation of POMC in vivo, since mutation or deletion of this site modestly reduces promoter activity in cultured corticotrophs (35). To summarize, activation of the JAK-STAT cascade activates several genes in the corticotroph cell by direct binding of activated STAT3 to the respective promoter regions of $P O M C$, Fos, and JunB. The transcription factors c-fos and JunB subsequently bind to the POMC promoter themselves. The direct and indirect mechanisms by which STATs induce POMC promoter activity constitute the molecular basis of the neuro-immunoendocrine regulation of corticotroph POMC expression by LIF and CRH (Figure 1).

In vivo, systemic LIF administration induces pituitary POMC transcription and ACTH secretion either alone or in synergy with $\mathrm{CRH}(37,38)$. Hypothalamic and pituitary LIF expression is induced by systemic inflammatory stimuli such as LPS (39), IL-1 $\beta$ (40), and CFA (41), as well as by local inflammatory stimuli, such as turpentine (41). LIF knockout animals show a corresponding decrease in hypothalamic-pituitary-adrenal (HPA) axis response to stress induced by immobilization $(38,42)$ or systemic inflammation (41). Conversely, pituitary-directed LIF overexpression in transgenic mice results in corticotroph hyperplasia and the usual sequelae of hypercortisonism, including obesity and failed dexamethasone suppression (43). Thus, LIF is required for both appropriate corticotroph development and function.

\section{SOCS-3 as a suppressor of STAT-dependent}

POMC expression

Corticotroph SOCS-3 coimmunoprecipitates with JAK2 (18). Furthermore, the overexpression of SOCS-3 inhibits LIF-induced phosphorylation of JAK2 (18), gp130 (33), and STAT3 (33). The physiological importance of this interaction is suggested by the phenotype of corticotroph cells stably overexpressing wild-type SOCS-3, which show significant inhibition of LIFinduced $P O M C$ promoter activation, $\mathrm{POMC}$ transcription, and ACTH secretion (33). Inhibition of LIFinduced STAT3 phosphorylation by SOCS-3 overexpression also blocks STAT3 protein binding to the bipartite STAT3-binding element in the POMC promoter (35). Consistent with the in vitro analysis described above, corticotroph SOCS-3 overexpression also abrogates LIF-induced c-fos and JunB expression (35). Thus, inhibition of LIF-induced STAT3 phosphorylation by SOCS-3 overexpression causes inhibition of corticotroph $P O M C$ promoter activity by direct negative regulation of STAT3 function, and indirectly

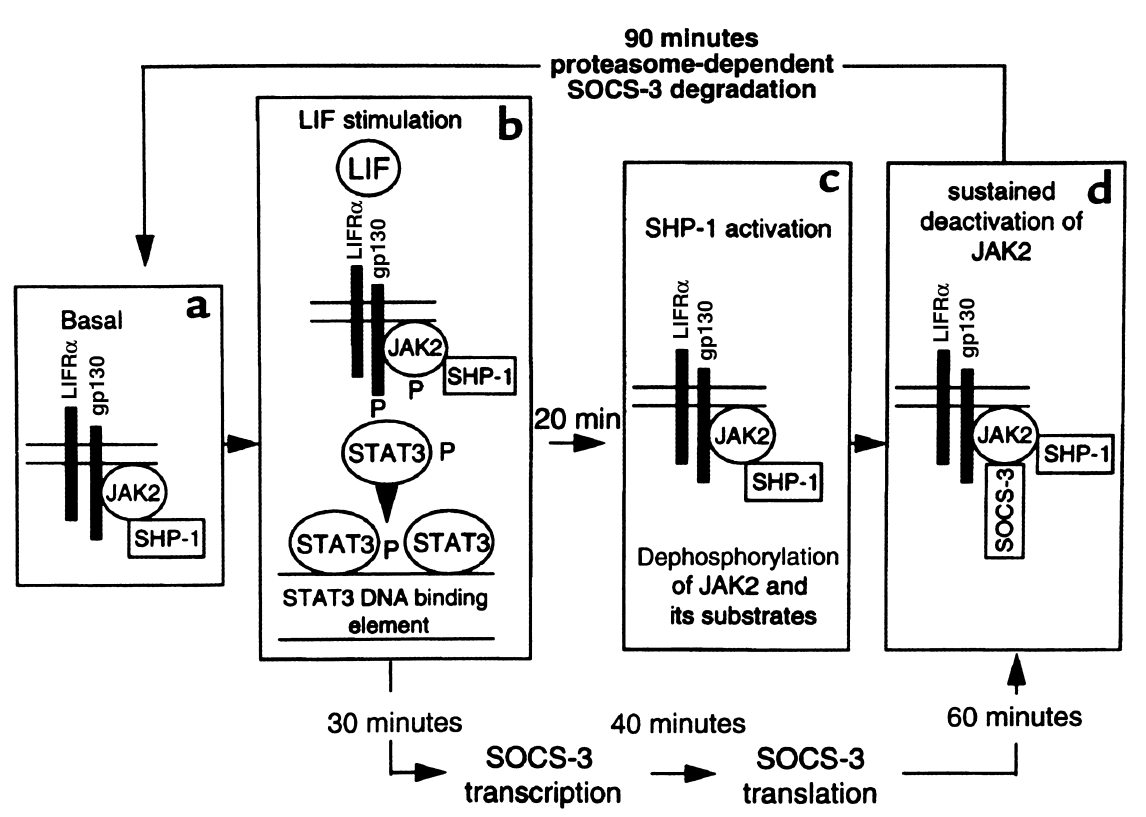

\section{Figure 2}

Inhibitory effects of SOCS-3 and SHP-1 on LIF-mediated gene expression. (a) The tyrosine kinase JAK2 and tyrosine phosphatase SHP-1 are constitutively expressed but remain inactive in the unstimulated corticotroph. In contrast, SOCS-3 expression in the unstimulated corticotroph is minimal. (b) LIF binding rapidly induces the LIF receptor (LIFR) and gp130 subunits to form a heterodimer receptor complex. Receptor complex formation leads to autophosphorylation of receptor-associated JAK2, followed by tyrosine phosphorylation of the receptor's cytoplasmic domain and recruitment of STAT proteins to the receptor complex. Subsequent tyrosine phosphorylation of STATs enables homo- or heterodimerization of STAT proteins. The dimerized STAT complexes translocate to the nucleus and bind to specific STAT-binding elements in the promoter region of various genes, among them SOCS3. (c) The tyrosine phosphatase SHP-1 is activated by LIF, showing maximum catalytic activity at 30 minutes. JAK2 and its substrates are dephosphorylated by SHP-1. Thus, SHP-1 is a constitutively expressed and rapidly activated inhibitor of JAK-STAT signaling in the corticotroph. (d) STAT-dependent SOCS3 gene expression is induced severalfold by LIF within 30 minutes. SOCS-3 protein associated with JAK2 is detectable 40-60 minutes after LIF stimulation. Association of SOCS-3 with JAK2 inhibits JAK2 activity. Thus, SOCS-3 inhibits JAK-STAT signaling in the corticotroph, its expression rapidly up- and downregulated by LIF and negative autoregulation of its own STAT-dependent gene expression. SOCS-3 is rapidly degraded by a proteasome-dependent pathway, allowing the corticotroph to return to its basal state, in which it can once again be activated by LIF or other gp 130 cytokines (see a). Reproduced from ref. 18 with permission. 
by suppression of the STAT-dependent transcription factors c-fos and JunB (Figure 1).

Murine corticotroph AtT-20 cells incubated with $0.1-10.0 \mathrm{ng} / \mathrm{ml}$ LIF exhibit rapid and potent stimulation of SOCS-3 mRNA expression, peaking at $30 \mathrm{~min}$ utes and persisting for as long as 8 hours $(28,33)$. The related inhibitors SOCS- 2 and CIS are not significantly induced under these circumstances. Interestingly, SOCS-3 mRNA is also induced, albeit at a lower magnitude, in corticotroph AtT-20 cells following incubation with IL-11 (28, 44), IL-6 (28), or IL-1 3 (33). Hence, in the corticotroph, the termination of signaling by multiple cytokines appears to depend on SOCS-3.

In vivo, the hypothalamus $(33,41,45,46)$ and pituitary $(33,41)$ of intact mice show very low basal expression of SOCS-3 mRNA but can induce SOCS- 3 expression within 30-60 minutes following systemic administration of LIF or IL-1 $\beta$ (33), respectively. Similarly, systemic administration of LPS endotoxin rapidly induces hypothalamic SOCS-3 expression (46). In other models of inflammation, subcutaneous injection of CFA or intramuscular administration of turpentine causes increased SOCS-3 mRNA pituitary expression, and to a lesser extent in the hypothalamus (41). This response in part reflects the action of an important STAT1/STAT3- binding element located in the SOCS3 promoter (28). Deletion or inactivating mutation of this binding element in the murine SOCS3 promoter almost completely abrogates promoter activity. Likewise, overexpression of dominant negative STAT3 mutants decreases LIF-induced SOCS3 promoter activity and gene expression (28), confirming that LIF-induced SOCS-3 expression in the corticotroph cell is critically STAT3-dependent. On the other hand, since SOCS-3 is a potent inhibitor of LIF-induced JAK-STAT signaling, SOCS-3 negatively regulates its own cytokine-induced expression at the mRNA level by a short intracellular inhibitory feedback loop (Figures 1 and 2).

Corticotroph SOCS-3 gene expression is also induced by cAMP analogues, such as pituitary adenylate cyclase-activating polypeptide (PACAP), $\mathrm{CRH}$, or epinephrine, which can act either alone or cooperatively with LIF (47). These effects on corticotroph SOCS-3 expression are protein kinase A-dependent (PKA-dependent), as overexpression of a dominant negative PKA isoform inhibits cAMP-mediated SOCS promoter activation (47). Following stimulation of AtT-20 cells with dibutyryl-cAMP, c-fos and JunB bind specifically to the AP-1 site in the SOCS3 promoter (47). Mutation of this AP-1 site inhibits dibutyrylcAMP-mediated SOCS3 promoter activation by approximately $40 \%$, consistent with evidence that dibutyryl-cAMP's effects on the SOCS3 promoter are mediated by several other elements in addition to the AP-1 site (47). As shown in Figure 2, corticotroph SOCS-3 protein expression is rapidly regulated and short-lived. LIF-induced SOCS-3 protein expression occurs as early as 20 minutes, peaking after $40-60$ minutes, and disappearing at 90 minutes. Preincubation of AtT-20 cells with the proteasome inhibitor LLnL resulted in LIF-induced SOCS-3 protein remaining detectable for as long as 180 minutes (18).
Deletion analysis of SOCS-3 has shed some light on the mechanism by which this protein suppresses JAKmediated LIF signaling. A truncated recombinant form of SOCS- 3 containing the pre-SH2 domain, the SH2 domain, and the SOCS-box motif inhibits LIF-induced $P O M C$ promoter activity. In contrast, constructs in which the SOCS-box motif or the pre-SH2 domain deletions are deleted are inactive (18). These results are consistent with other studies, demonstrating the essential roles of pre-SH2 and $\mathrm{SH} 2$ domains of SOCS-3 and SOCS-1, respectively, for suppression of the JAK-STAT cascade. The SOCS-box motif alone is sufficient to inhibit corticotroph LIF signaling by SOCS-3 (18). However, the requirement for the SOCS-box and other motifs for the suppressive action of SOCS proteins is controversial $(19,20)$ and requires further investigation.

SOCS-3 as a negative regulator of leptin signaling SOCS-3 is a candidate leptin resistance factor. Overexpression of SOCS-3 results in inhibition of leptininduced tyrosine phosphorylation of JAK2 (23). Mutational analysis shows that Tyr985, which is essential for recruitment of SHP-2 $(48)$ and SOCS-3 $(25,26)$ to the intracellular domain of the long leptin receptor isoform, is not required for STAT3 signaling. Binding of SOCS-3 to this site is nevertheless required for the inhibition of leptin signaling (25), at least when SOCS-3 is present at normal levels; higher, possibly supraphysiological concentrations of SOCS-3 may also inhibit leptin signaling by direct association with JAK2 $(23,25)$. In vitro incubation with leptin induces expression of SOCS- 3 but not SOCS-1, SOCS-2, or CIS (23).

Following systemic leptin administration, leptin-deficient $o b / o b$ mice (23) as well as Wistar rats show an increase in hypothalamic SOCS-3 expression. Several other in vivo findings suggest that SOCS- 3 reduces leptin sensitivity. Hypothalamic SOCS-3 expression is increased in the lethal yellow (Ay/a) mouse, which is subject to both hyperleptinemia and leptin resistance (45). Hypothalamic SOCS-3 expression also increased in 18-month-old versus 2-month-old rats, the former exhibiting relative leptin resistance (49).

\section{SOCS-3 as a negative regulator of $\mathrm{GH}$ signaling}

GH rapidly and potently induces hepatic SOCS-3 expression $(13,50)$, suggesting that signaling by this factor, too, may be terminated by SOCS-3, and raising the possibility that heightened expression of this regulator could lead to the GH resistance seen in chronic illness or inflammatory states. Induction of hepatic SOCS-1 following GH is weak (13) or undetectable (50); CIS and SOCS-2 are induced in the liver, although with much slower kinetics than SOCS-3 $(13,50)$.

Overexpression of SOCS-3 and SOCS-1 (but not of CIS or SOCS-2; refs. 13-16, 51) strongly inhibits GH (13-15) and prolactin (16) signaling. Interestingly, high SOCS-2 expression has been reported to superinduce GH signaling $(13,14)$. SOCS-1-inhibited GH and prolactin signaling is restored in a concentrationdependent manner by SOCS-2 coexpression $(14,16)$. The same phenomenon is not observed during coexpression of SOCS-3 and SOCS-2 $(14,16)$. Thus, the 
rapidly induced SOCS- 3 and SOCS- 1 genes might switch off GH signaling, which could then be restored by later induction of SOCS-2, which partially antagonizes SOCS-1 action.

Bacterial endotoxin and IL-1 $\beta$ induce hepatic SOCS-3 gene expression $(52,53)$ and inhibit GH signaling. GH resistance, characteristic of several inflammatory states, could thus be mediated by hepatic SOCS-3 expression, induced by endotoxin and various inflammatory cytokines, e.g., IL-6, IL- $1 \beta$, and TNF- $\alpha(52,53)$. SOCS-3 subsequently mediates $\mathrm{GH}$ resistance by inhibition of intracellular GH signaling. The stunted growth observed in children with chronic illness is likely caused by GH resistance mediated by cytokine-induced intracellular inhibition of GH action.

\section{Summary}

SOCS-3 is a potent inhibitor of the JAK-STAT signaling cascade, negatively regulating signal transduction of a variety of cytokines, including gp130 cytokines, leptin, and GH.

Several gp130 cytokines are neuro-immune modulators of HPA axis function. LIF action on corticotroph cell function is well characterized and has been demonstrated to be important for the HPA axis response. LIF upregulates POMC gene expression as well as SOCS-3 gene expression by STAT-dependent mechanisms. Following LIF stimulation, corticotroph SOCS-3 expression is rapidly upregulated. Cellular SOCS-3 expression is tightly controlled by negative autoregulation of its own STAT-dependent promoter activity as well as short protein half-life enabling rapid "on" and "off" mechanisms, subserving corticotroph plasticity toward various neuro-immune stimuli. SOCS-3 also contributes to central leptin resistance and hepatic GH resistance. Thus, SOCS-3 plays a critical role in integrating the neuro-immunoendocrine interface in the HPA axis as well as other neuro-immunoendocrine circuits.

1. Imada, K., and Leonard, W.J. 2000. The Jak-STAT pathway. Mol. Immunol. 37:1-11

2. Auernhammer, C.J., and Melmed, S. 2000. Leukemia-inhibitory-factor: neuroimmmune modulator of endocrine function. Endocr. Rev. 21:313-345.

3. Starr, R., et al. 1997. A family of cytokine-inducible inhibitors of signalling. Nature. 387:917-921.

4. Endo, T.A., et al. 1997. A new protein containing an SH2 domain that inhibits JAK kinases. Nature. 387:921-924.

5. Hilton, D.J., et al. 1998. Twenty proteins containing a C-terminal SOCS box form five structural classes. Proc. Natl. Acad. Sci. USA. 95:114-119.

6. Ohya, K., et al. 1997. SOCS-1/JAB/SSI-1 can bind to and suppress Tec protein-tyrosine kinase. J. Biol. Chem. 272:27178-27182.

7. Matsumoto, A., et al. 1997. CIS, a cytokine inducible SH2 protein, is a target of the Jak-STAT5 pathway and modulates STAT5 activation. Blood. 89:3148-3154.

8. Artz, E. 2001. gp130 cytokine signaling in the pituitary gland: a paradigm for cytokine-neuro-endocrine pathways. J. Clin. Invest. 108:1729-1733.

9. Song, M.M., and Shuai, K. 1998.The suppressor of cytokine signaling (SOCS) 1 and SOCS3 but not SOCS2 proteins inhibit interferon-mediated antiviral and antiproliferative activities. J. Biol. Chem. 273:35056-35062.

10. Nicholson, S.E., et al. 1999. Mutational analyses of the SOCS proteins suggest a dual domain requirement but distinct mechanisms for inhibition of LIF and IL-6 signal transduction. EMBO J. 18:375-385.

11. Duval, D., Reinhardt, B., Kedinger, C., and Boeuf, H. 2000. Role of suppressors of cytokine signaling (Socs) in leukemia inhibitory factor (LIF)dependent embryonic stem cell survival. FASEB J. 14:1577-1584.

12. Hamanaka, I., et al. 2001. Induction of JAB/SOCS-1/SSI-1 and
CIS3/SOCS-3/SSI-3 is involved in gp130 resistance in cardiovascular system in rat treated with cardiotrophin-1 in vivo. Circ. Res. 88:727-732.

13. Adams, T.E., et al. 1998. Growth hormone preferentially induces the rapid, transient expression of SOCS-3, a novel inhibitor of cytokine receptor signaling. J. Biol. Chem. 273:1285-1287.

14. Favre, H., Benhamou, A., Finidori, J., Kelly, P.A., and Edery, M. 1999. Dual effects of suppressor of cytokine signaling (SOCS-2) on growth hormone signal transduction. FEBS Lett. 453:63-66.

15. Ram, P.A., and Waxman, D.J. 1999. SOCS/CIS protein inhibition of growth hormone-stimulated STAT5 signaling by multiple mechanisms. J. Biol. Chem. 274:35553-35561.

16. Pezet, A., Favre, H., Kelly, P.A., and Edery, M. 1999. Inhibition and restoration of prolactin signal transduction by suppressors of cytokine signaling. J. Biol. Chem. 274:24497-24502.

17. Yasukawa, H., et al. 1999. The JAK-binding protein JAB inhibits Janus tyrosine kinase activity through binding in the activation loop. EMBOJ. 18:1309-1320.

18. Bousquet, C., Susini, C., and Melmed, S. 1999. Inhibitory roles for SHP1 and SOCS-3 following pituitary proopiomelanocortin induction by leukemia inhibitory factor. J. Clin. Invest. 104:1277-1285.

19. Zhang, J.G., et al. 1999. The conserved SOCS box motif in suppressors of cytokine signaling binds to elongins B and C and may couple bound proteins to proteasomal degradation. Proc. Natl. Acad. Sci. USA. 96:2071-2076.

20. Okabe, S., et al. 1999. Thrombopoietin induces an SH2-containing protein, CIS1, which binds to $\mathrm{Mpl}$ : involvement of the ubiquitin proteosome pathway. Exp. Hematol. 27:1542-1547.

21. Siewert, E., Muller-Esterl, W., Starr, R., Heinrich, P.C., and Schaper, F. 1999. Different protein turnover of interleukin-6-type cytokine signalling components. Eur. J. Biochem. 265:251-257.

22. Sporri, B., Kovanen, P.E., Sasaki, A., Yoshimura, A., and Leonard, W.J. 2001. JAB/SOCS1/SSI-1 is an interleukin-2-induced inhibitor of IL-2 signaling. Blood. 97:221-226.

23. Bjorbaek, C., El-Haschimi, K., Frantz, J.D., and Flier, J.S. 1999. The role of SOCS-3 in leptin signaling and leptin resistance. J. Biol. Chem. 274:30059-30065.

24. Nicholson, S.E., et al. 2000. Suppressor of cytokine signaling-3 preferentially binds to the SHP-2-binding site on the shared cytokine receptor subunit gp130. Proc. Natl. Acad. Sci. USA. 97:6493-6498.

25. Bjorbak, C., et al. 2000. SOCS3 mediates feedback inhibition of the leptin receptor via Tyr985. J. Biol. Chem. 275:40649-40657.

26. Eyckerman, S., Broekaert, D., Verhee, A., Vandekerckhove, J., and Tavernier, J. 2000. Identification of the Y985 and Y1077 motifs as SOCS3 recruitment sites in the murine leptin receptor. FEBS Lett. 486:33-37.

27. Verdier, F., et al. 1998. A sequence of the CIS gene promoter interacts preferentially with two associated STAT5A dimers: a distinct biochemical difference between STAT5A and STAT5B. Mol. Cell. Biol. 18:5852-5860.

28. Auernhammer, C.J., Bousquet, C., and Melmed, S. 1999. Autoregulation of pituitary corticotroph SOCS-3 expression: characterization of the murine SOCS-3 promoter. Proc. Natl. Acad. Sci. USA. 96:6964-6969.

29. Wang, D., Stravopodis, D., Teglund, S., Kitazawa, J., and Ihle, J.N. 1996. Naturally occurring dominant negative variants of Stat5. Mol. Cell. Biol. 16:6141-6148.

30. Akita, S., et al. 1995. Human and murine pituitary expression of leukemia inhibitory factor. Novel intrapituitary regulation of adrenocorticotropin hormone synthesis and secretion. J. Clin. Invest. 95:1288-1298.

31. Ray, D.W., Ren, S.G., and Melmed, S. 1996. Leukemia inhibitory factor (LIF) stimulates proopiomelanocortin (POMC) expression in a corticotroph cell line. Role of STAT pathway. J. Clin. Invest. 97:1852-1859.

32. Shimon, I., Yan, X., Ray, D.W., and Melmed, S. 1997. Cytokine-dependent gp130 receptor subunit regulates human fetal pituitary adrenocorticotropin hormone and growth hormone secretion. J. Clin. Invest. 100:357-363.

33. Auernhammer, C.J., Chesnokova, V., Bousquet, C., and Melmed, S. 1998. Pituitary corticotroph SOCS-3: novel intracellular regulation of leukemia-inhibitory factor-mediated proopiomelanocortin gene expression and adrenocorticotropin secretion. Mol. Endocrinol. 12:954-961.

34. Bousquet, C., and Melmed, S. 1999. Critical role for STAT3 in murine pituitary adrenocorticotropin hormone leukemia inhibitory factor signaling. J. Biol. Chem. 274:10723-10730.

35. Bousquet, C., Zatelli, M.C., and Melmed, S. 2000. Direct regulation of pituitary proopiomelanocortin by STAT3 provides a novel mechanism for immuno-neuroendocrine interfacing. J. Clin. Invest. 106:1417-1425.

36. Bousquet, C., Ray, D.W., and Melmed, S. 1997. A common pro-opiomelanocortin-binding element mediates leukemia inhibitory factor and corticotropin-releasing hormone transcriptional synergy. J. Biol. Chem. 272:10551-10557.

37. Akita, S., Conn, P.M., and Melmed, S. 1996. Leukemia inhibitory factor (LIF) induces acute adrenocorticotrophic hormone (ACTH) secretion in fetal rhesus macaque primates: a novel dynamic test of pituitary function. J. Clin. Endocrinol. Metab. 81:4170-4178. 
38. Chesnokova, V., Auernhammer, C.J., and Melmed, S. 1998. Murine leukemia inhibitory factor gene disruption attenuates the hypothalamopituitary-adrenal axis stress response. Endocrinology. 139:2209-2216.

39. Wang, Z., Ren, S.G., and Melmed, S. 1996. Hypothalamic and pituitary leukemia inhibitory factor gene expression in vivo: a novel endotoxininducible neuro-endocrine interface. Endocrinology. 137:2947-2953.

40. Auernhammer, C.J., Chesnokova, V., and Melmed, S. 1998. Leukemia inhibitory factor modulates interleukin-1beta-induced activation of the hypothalamo-pituitary-adrenal axis. Endocrinology. 139:2201-2208.

41. Chesnokova, V., and Melmed, S. 2000. Leukemia inhibitory factor mediates the hypothalamic pituitary adrenal axis response to inflammation. Endocrinology. 141:4032-4040.

42. Akita, S., Malkin, J., and Melmed, S. 1996. Disrupted murine leukemia inhibitory factor (LIF) gene attenuates adrenocorticotropic hormone (ACTH) secretion. Endocrinology. 137:3140-3143.

43. Yano, H., Readhead, C., Nakashima, M., Ren, S.G., and Melmed, S. 1998. Pituitary-directed leukemia inhibitory factor transgene causes Cushing's syndrome: neuro-immune-endocrine modulation of pituitary development. Mol. Endocrinol. 12:1708-1720.

44. Auernhammer, C.J., and Melmed, S. 1999. Interleukin-11 stimulates POMC gene expression and ACTH secretion in corticotroph cells: evidence for a redundant cytokine network in the HPA axis. Endocrinology. 140:1559-1566.

45. Bjorbaek, C., Elmquist, J.K., Frantz, J.D., Shoelson, S.E., and Flier, J.S. 1998. Identification of SOCS-3 as a potential mediator of central leptin resistance. Mol. Cell. 1:619-625.
46. Lebel, E., Vallieres, L., and Rivest, S. 2000. Selective involvement of interleukin- 6 in the transcriptional activation of the suppressor of cytokine signaling-3 in the brain during systemic immune challenges. Endocrinology. 141:3749-3763.

47. Bousquet, C., Chesnokova, V., Kariagina, A., Ferrand, A., and Melmed, S 2001. cAMP neuropeptide agonists induce pituitary suppressor of cytokine signaling-3: novel negative feedback mechanism for corticotroph cytokine action. Mol. Endocrinol. 15:1880-1890.

48. Banks, A.S., Davis, S.M., Bates, S.H., and Myers, M.G., Jr. 2000. Activation of downstream signals by the long form of the leptin receptor. J. Biol. Chem. 275:14563-14572.

49. Wang, Z.W., et al. 2001. The role of leptin resistance in the lipid abnormalities of aging. FASEB J. 15:108-114.

50. Davey, H.W., McLachlan, M.J., Wilkins, R.J., Hilton, D.J., and Adams, T.E. 1999. STAT5b mediates the GH-induced expression of SOCS-2 and SOCS-3 mRNA in the liver. Mol. Cell. Endocrinol. 158:111-116.

51. Karlsson, H., Gustafsson, J.A., and Mode, A. 1999. Cis desensitizes GH induced Stat5 signaling in rat liver cells. Mol. Cell. Endocrinol. 154:37-43.

52. Mao, Y., et al. 1999. Endotoxin-induced inhibition of growth hormone receptor signaling in rat liver in vivo. Endocrinology. 140:5505-5515.

53. Colson, A., Le Cam, A., Maiter, D., Edery, M., and Thissen, J.P. 2000. Potentiation of growth hormone-induced liver suppressors of cytokine signaling messenger ribonucleic acid by cytokines. Endocrinology. 141:3687-3695 